\title{
Risk of neutropenia among clozapine users and non-users: results from 5,847 patients
}

\author{
André Akira Sueno Goldani, ${ }^{1,2,3,4 *}$ (iD Francisco Diego Rabelo-da-Ponte, ${ }^{1,2,3,4 \star}$ (iD Jacson Gabriel \\ Feiten, ${ }^{1,2,3,4 *}$ iD Maria Ines R. Lobato, ${ }^{4}$ iD Paulo S. Belmonte-de-Abreu, ${ }^{4}$ id Clarissa S. Gama ${ }^{1,2,3,4}$ iD \\ ${ }^{1}$ Laboratório de Psiquiatria Molecular, Centro de Pesquisa Experimental, Hospital de Clínicas de Porto Alegre (HCPA), Universidade Federal \\ do Rio Grande do Sul (UFRGS), Porto Alegre, RS, Brazil. ${ }^{2}$ Laboratório de Psiquiatria Molecular, Centro de Pesquisa Clínica, HCPA, UFRGS, \\ Porto Alegre, RS, Brazil. ${ }^{3}$ Instituto Nacional de Ciência e Tecnologia Translacional em Medicina, UFRGS, Porto Alegre, RS, Brazil. ${ }^{4}$ Programa \\ de Pós-Graduação em Psiquiatria e Ciências do Comportamento, Departamento de Psiquiatria, Faculdade de Medicina, UFRGS, Porto \\ Alegre, RS, Brazil. * These authors have contributed equally to this manuscript.
}

\begin{abstract}
Objective: Clozapine is underprescribed due to neutropenia risk. Blood tests every 3 months in those on continuous treatment for $>1$ year who have never had an absolute neutrophil count (ANC) $<2,000 / \mu \mathrm{L}$ has been proposed as a monitoring strategy; however, there are no South American data to support this recommendation. This study sought to investigate whether clozapine use and other variables could explain the occurrence of $\mathrm{ANC}<1,000 / \mu \mathrm{L}$ in patients with severe mental disorders. Methods: A total of 5,847 subjects were included, 1,038 on clozapine. We performed a Cox regression considering the outcome as $\mathrm{ANC}<1,000 / \mu \mathrm{L}$ at any time point. Predictors were sex, age, ethnicity, clozapine use, ANC $>2,000 / \mu \mathrm{L}$ during the first year of blood monitoring, and presence of a severe medical condition.

Results: In the Cox regression model, ethnicity (white) (hazard ratio [HR] 0.53; 95\% $\mathrm{Cl} 0.29-0.99$, $\mathrm{p}<0.05)$ and ANC $>2,000 / \mu \mathrm{L}(\mathrm{HR} 0.04 ; 95 \% \mathrm{Cl} 0.01-0.10, \mathrm{p}<0.001)$ were protective factors, while presence of a severe medical condition (HR 69.35; 95\% Cl 37.45-128.44, $\mathrm{p}<0.001$ ) was a risk factor for ANC $<1,000 / \mu \mathrm{L}$. Other variables were not significant, including clozapine use $(\mathrm{HR} 1.33 ; 95 \% \mathrm{Cl}$ $0.74-2.39, \mathrm{p}>0.05)$.

Conclusions: These findings suggest that clozapine does not increase the risk of neutropenia in subjects with ANC $>2,000 / \mu \mathrm{L}$ during the first year of use and in the absence of a severe medical condition. These results could help guide clinical and public-health decisions regarding clozapine blood monitoring guidelines.
\end{abstract}

Keywords: Clozapine; neutropenia; hematological monitoring; hemogram; absolute neutrophil count

\section{Introduction}

Clozapine, an atypical antipsychotic, distinguishes itself among its class for higher efficacy in several chronic mental health disorders, such as schizophrenia, bipolar disorder, and Parkinson's disease psychosis. ${ }^{1-3}$ Particularly in schizophrenia, its use is associated with better outcomes in treatment resistance, with lower suicidality rates and lower long-term mortality. ${ }^{4-6}$ However, clozapine is underused due to prescribers' concern for the risk, albeit rare, of developing neutropenia and subsequent agranulocytosis. $^{7}$

Initially made available in the 1970s, clozapine was withdrawn after the report of eight fatal cases of agranulocytosis. $^{8}$ It was later returned to market under strict absolute neutrophil count (ANC) monitoring, but there are discrepancies among countries regarding

Correspondence: Clarissa S. Gama, Centro de Pesquisa Experimental, Hospital de Clínicas de Porto Alegre, Universidade Federal do Rio Grande do Sul, Rua Ramiro Barcelos, 2350, Prédio Anexo, CEP 90035-903, Porto Alegre, RS, Brazil.

E-mail: cgama@hcpa.edu.br

Submitted Jan 21 2021, accepted Jul 10 2021, Epub Oct 292021. required surveillance regimens. ${ }^{9,10}$ Currently, in the United States, the prescribed regimen involved weekly ANC monitoring during the first 6 months; every other week for the second 6 months; and every 4 weeks after 1 year of treatment, with monthly monitoring thereafter for the duration of treatment (Clozapine REMS Program ${ }^{11}$ ). In Brazil and several other countries, the stipulated regimen is weekly during the first 18 weeks and monthly thereafter. ${ }^{10}$ In some settings, the prescription of clozapine is further withheld on account of this burdensome long-term monitoring. ${ }^{12}$

Recently, the literature has been challenging the requirement of monthly ANC follow-up after the first 18 weeks. In a meta-analysis of 425,774 individuals on clozapine therapy, neutropenia-related mortality was rare and neutropenia incidence rates were low after 1 year of treatment. ${ }^{13}$ In another report, the same authors did not

How to cite this article: Goldani AAS, Rabelo-da-Ponte FD, Feiten JG, Lobato MIR, Belmonte-de-Abreu PS, Gama CS. Risk of neutropenia among clozapine users and non-users: results from 5,847 patients. Braz J Psychiatry. 2022;44:21-25. http://dx.doi.org/ 10.1590/1516-4446-2021-1765 
observe a difference in incident neutropenia between clozapine users and users of other atypical antipsychotics. ${ }^{14}$ Moreover, reports in Japan and Chile corroborate low long-term incidence rates of neutropenia, with $90.2 \%$ occurring in the first year and $80 \%$ in the first 18 weeks of treatment respectively. ${ }^{15,16}$

Furthermore, a more flexible long-term monitoring approach has been debated in order to prevent unnecessary exposure of at-risk patients during the severe acute respiratory syndrome coronavirus 2 (SARS-CoV-2) pandemic. The latest consensus is to monitor every 3 months patients who fulfill the following criteria: continuous treatment for $>1$ year; never had an ANC $<2,000 / \mu \mathrm{L}$ (or $<1,500 / \mu \mathrm{L}$ if history of benign ethnic neutropenia); no safe or practical access to ANC testing. ${ }^{17}$ The Latin American population is one of the most ethnically diverse worldwide, because of miscegenation of native groups with European, African, and Asian migrants. There are no large-scale studies describing the characteristics of the Brazilian population regarding clozapine blood monitoring, and few reports in other Latin American countries. $^{16,18}$ Thus, our general aim was to investigate whether clozapine use and other variables could explain the occurrence of ANC $<1,000$ in a large sample of patients with severe mental disorders in several settings of a general university hospital in Porto Alegre, the capital of the southernmost state of Brazil.

\section{Methods}

\section{Participants}

We included 5,847 patients with severe mental disorders in several settings (i.e., inpatient and outpatient clinics) of a tertiary mental health referral service, Hospital de Clínicas de Porto Alegre (HCPA). Participants who had at least one ANC value entered into their electronic medical record (EMR) during the years 2005-2020 were included.

Participants' information was obtained from the HCPA EMR. The inpatient subgroup is characterized by adult patients (age $>18$ years) in acute brief hospitalization without a substance use disorder as the main diagnosis. The outpatient subgroup mainly comprised patients diagnosed with a chronic psychotic disorder (i.e., schizophrenia), bipolar disorder, or other major functional impairment due to psychiatric disorders. Patients who were prescribed clozapine at least once during their follow-up at HCPA were identified as clozapine users. Demographic characteristics were collected by the hospital's administrative staff members, who are instructed to check official identification documents and enter ethnicity as self-declared (white non-Hispanic, black, native Brazilian, Asian, or other). The population of the state of Rio Grande do Sul, the southernmost state of Brazil, is composed mainly of white non-Hispanics.

\section{Data analysis}

We reported descriptive analyses as mean (standard deviation $[S D]$ ), median (interquartile range [IQR]), or absolute and relative frequencies, as appropriate. Participants were separated into two groups for descriptive analyses: clozapine users and non-users. The ShapiroWilk test was used to assess normality of data distribution, the chi-square test to analyze categorical data, the MannWhitney $U$ test to analyze nonparametric continuous variables, and Student's $t$ test for parametric continuous variables. We performed a multivariable Cox proportionalhazards regression to assess independent risk factors for moderate and severe neutropenia (ANC $<1,000 / \mu \mathrm{L}$ ), including sex, age (years), ethnicity, clozapine use, ANC above $2,000 / \mu \mathrm{L}$ during the first year of clozapine blood monitoring records or beyond, and the presence of a severe medical condition (i.e., human immunodeficiency virus [HIV], hepatitis $\mathrm{C}$ virus [HCV], cancer). Hazard ratios (HR) with $95 \%$ confidence intervals $(95 \% \mathrm{Cls})$ and $\mathrm{p}$-values from a Wald test were reported. A two-tailed $p$-value $<0.05$ was considered as significant. The statistical analysis was performed using $R$ software (version 4.0.2), RStudio (version 1.3), and the 'survival' R package (version 3.2.7) for Cox regression.

\section{Ethics statement}

Our study was approved by the local ethics committee and conducted in accordance with the Declaration of Helsinki. Informed consent was waived since there was no more than minimal risk to privacy or harm of the individuals studied.

\section{Results}

Sociodemographic and clinical features are described in Table 1. The clozapine user and non-user groups differed in terms of sex, age, ethnicity, and educational status. Additionally, clozapine users underwent more blood tests and, consequently, had a higher number of follow-up days than non-clozapine users. Most individuals on clozapine had a diagnosis of schizophrenia or a severe mental condition (diagnosis not reported). The exact date of clozapine initiation was assumed from the first available ANC of patients that were identified to be on clozapine. In our sample of 1,038 clozapine users, during a median follow-up of 1,071 days (36 months), the absolute risk of severe neutropenia was $0.77 \%($ ANC $<500 / \mu \mathrm{L})$, and the risk of overall neutropenia (ANC $<1,500 / \mu \mathrm{L}$ ) was $5.3 \%$. There was no reported mortality due to neutropenia in either clozapine users or non-users.

In the multivariable Cox proportional-hazards regression model (Wald test [6] $=237.6, p<0.001$ ), we found that ethnicity (white) (HR 0.53 [95\% Cl 0.29-0.99], p < 0.05 ), ANC above $2,000 / \mu \mathrm{L}$ during the first year of clozapine blood monitoring records or beyond (HR 0.04 [95\% $\mathrm{Cl} 0.01-0.10], \mathrm{p}<0.001)$, and the presence of a severe medical condition (HR $69.35[95 \% \mathrm{Cl}$ 37.45128.44], $\mathrm{p}<0.001$ ) were significantly associated with development of moderate and severe neutropenia (ANC $<1,000 / \mu \mathrm{L}$ ), the first two as protective factors and the latter as a risk factor (Figure 1). Other variables, including clozapine use, did not show any significant effect on outcome. 
Table 1 Sociodemographic and clinical variables of inpatients and outpatients with severe mental illness who underwent at least one ANC measurement from 2005 to 2020

\begin{tabular}{|c|c|c|c|}
\hline Sociodemographic and clinical variables & No clozapine $(n=4,809)$ & Clozapine $(n=1,038)$ & p-value \\
\hline Gender: male & $2,098(43.6)$ & $672(64.7)$ & $<0.001^{\dagger}$ \\
\hline Age in years, mean (SD) & $43.5(16.3)$ & $40.1(14.7)$ & $<0.001^{\text {末 }}$ \\
\hline Ethnicity: white non-Hispanic & $4,116(85.5)$ & $921(88.7)$ & $0.012^{\dagger}$ \\
\hline \multicolumn{4}{|l|}{ Education } \\
\hline Primary & $2,196(45.6)$ & $466(44.8)$ & \multirow[t]{4}{*}{$<0.001^{+}$} \\
\hline Secondary & $1,411(29.3)$ & $320(30.8)$ & \\
\hline Tertiary & $981(20.3)$ & $208(20.0)$ & \\
\hline Missing & $221(4.5)$ & $44(4.2)$ & \\
\hline Number of blood tests, median (IQR) & $2(1-3)$ & $17(6-57)$ & $<0.001^{\S}$ \\
\hline Follow-up days, median (IQR) & $3(0-122)$ & $1,071(68-3,283)$ & $<0.001^{\S}$ \\
\hline \multicolumn{4}{|l|}{ Diagnosis } \\
\hline Schizophrenia & $451(9.3)$ & $452(43.5)$ & \multirow[t]{3}{*}{$<0.001^{\dagger}$} \\
\hline Bipolar disorder & $631(13.1)$ & $100(9.6)$ & \\
\hline Severe mental illness (not otherwise specified) & $3,727(77.5)$ & $486(46.8)$ & \\
\hline Documented antipsychotic use (\%) & $1,891(39.3)$ & $1,048(100,00)$ & \\
\hline Absolute risk ANC $<2,000 / \mu \mathrm{L}$ & 10.67 & 13.97 & \\
\hline Absolute risk $\mathrm{ANC}<1,500 / \mu \mathrm{L}$ & 4.24 & 5.30 & \\
\hline Absolute risk $\mathrm{ANC}<1,000 / \mu \mathrm{L}$ & 0.79 & 1.64 & \\
\hline Absolute risk $\mathrm{ANC}<500 / \mu \mathrm{L}$ & 0.17 & 0.77 & \\
\hline
\end{tabular}

Data presented as $\mathrm{n}(\%)$, unless otherwise specified.

ANC = absolute neutrophil count; IQR = interquartile range; SD = standard deviation.

Chi-square test.

\$ Student $t$ test.

$\S$ Mann-Whitney $U$.

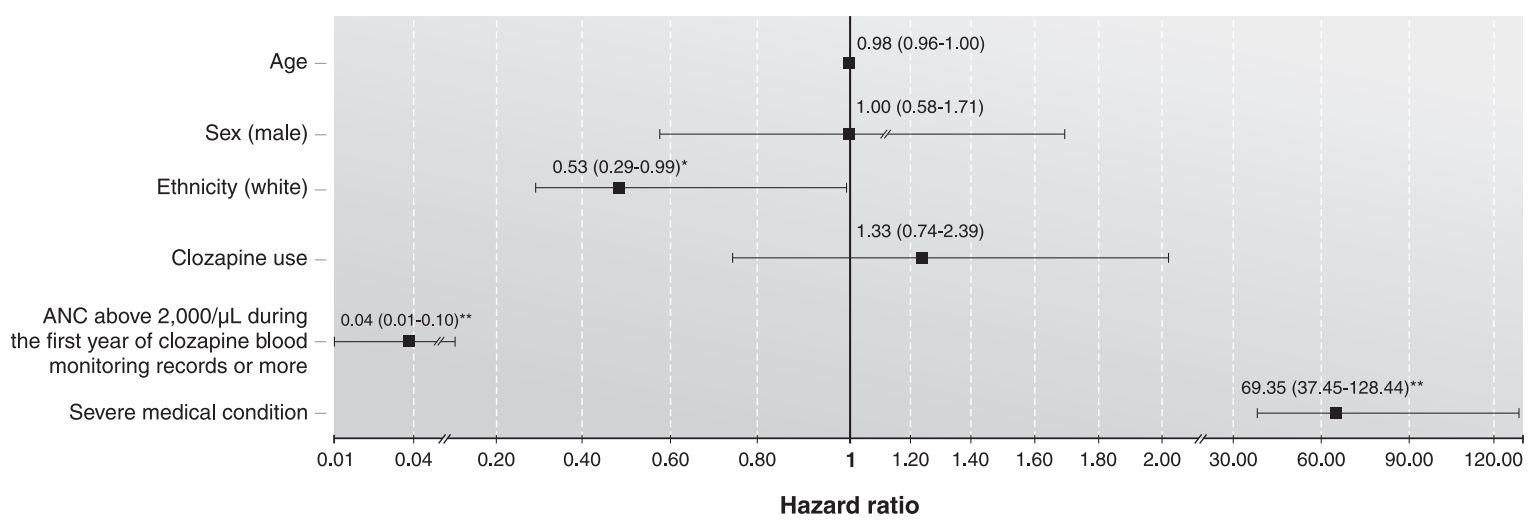

Figure 1 Multivariate Cox proportional-hazards regression model to assess independent risk factors for moderate and severe neutropenia (absolute neutrophil count $[\mathrm{ANC}]<1,000 / \mu \mathrm{L})$. Data are expressed as hazard ratio (HR) and $95 \%$ confidence interval $(95 \% \mathrm{Cl})$. Bars represent $95 \% \mathrm{Cl}$. Square is the value of hazard ratio. ${ }^{*} \mathrm{p}<0.05 ;{ }^{* \star} p<0.001$.

\section{Discussion}

Brazil is a large, populous country without a centralized registry for clozapine use. In the last 5 years, other South American countries have reported neutropenia prevalence and incidence in their national registries of clozapine use (i.e., Chile, Argentina, and Colombia). ${ }^{16,18,19}$ However, our study is the first to assess the risk of neutropenia in a severe mental illness sample with both clozapine and non-clozapine users in a treatment-as-usual setting in the subcontinent. Our analysis corroborates a recent meta-analysis which demonstrated non-different neutropenia rates when clozapine was compared to other antipsychotics in randomized clinical trials. ${ }^{14}$ In our study, despite both groups not being statistically matched as in a casecontrol design, pooled analysis is still relevant, since it comprises patients on an array of psychiatric medications in a naturalistic context, allowing higher external validity. 
Our findings demonstrated that ANC above 2,000/ $\mu \mathrm{L}$ during the first year of clozapine blood monitoring records or beyond was a significantly protective factor (HR 0.04; $95 \% \mathrm{Cl} 0.01-0.10$ ) for neutropenia risk $<1,000 / \mu \mathrm{L}$ at any time in a population with severe mental illness, regardless of clozapine use. The rationale of investigating this subgroup of patients is the largely reported low risk of neutropenia after the first year of clozapine use. Therefore, our results substantiate the recent recommendation for more flexible ANC monitoring strategies during the SARS-CoV-2 pandemic for clozapine users with a stable ANC above 2,000/ $\mu \mathrm{L}$ for at least 1 year. ${ }^{17}$ There are presumably other advantages of a lower frequency of ANC tests for clozapine users, such as lower COVID-19 exposure and a lighter resource burden for healthcare systems. Also, it could facilitate clozapine prescription for patients in whom it would be otherwise withheld due to safety and monitoring feasibility concerns. ${ }^{12}$ Ultimately, this could contribute to a higher proportion of treatmentresistant schizophrenia patients being adequately treated, thus improving symptom control and life expectancy. ${ }^{20}$

However, within the subgroup of patients who developed neutropenia, the presence of a medical comorbidity had a significantly elevated HR for this outcome. This is interpreted as the co-occurrence of lower neutrophil counts due to certain medical conditions and/or their respective treatments, such as malignant neoplasia, AIDS, and active viral hepatitis. Consequently, patients presenting with a potential neutrophil-lowering condition or treatment should undergo a more stringent monitoring regimen, regardless of clozapine use.

Another significant protective factor for neutropenia $<1,000 / \mu \mathrm{L}$ was white non-Hispanic ethnicity (HR 0.53; $95 \% \mathrm{Cl} 0.29-0.99$ ). This finding could be explained by the higher incidence of benign ethnic neutropenia in the African American population, ${ }^{21}$ and is thus aligned with Centers for Disease Control and Prevention (CDC) monitoring guidelines with a lower diagnostic criterion for neutropenia.

Sex and age were not associated with a higher risk of neutropenia. A major difference between clozapine users and non-users is the longer follow-up of the former compared to the latter. This discrepancy is probably due to the differential monitoring regimen for clozapine users, as evidenced also by the higher number of blood tests in the clozapine group.

Although not the main objective of this study, we also reported on overall absolute risk of neutropenia. In our sample of 1,038 clozapine users, during a median followup 1,071 days (36 months), the absolute risk of severe neutropenia (ANC $<500 / \mu \mathrm{L}$ ) was $0.77 \%$, similar to those reported by a meta-analysis and a naturalistic study (respectively 0.9 and $0.61 \%$ ). ${ }^{13,16}$ However, the absolute risk of overall neutropenia (ANC $<1,500 / \mu \mathrm{L}$ ) was $5.3 \%$, higher than reported in previous studies. ${ }^{13,16}$ The higher overall neutropenia rate could be explained by a number of reasons. First, our sample was composed exclusively of patients seen in tertiary care, derived in part from acute psychiatric inpatient settings. Although some clozapine users might initiate the medication in this treatment setting, it is usually reserved for more severe and acute condition, which are likely to involve higher use of concomitant medications and comorbid non-psychiatric conditions. Age was not a significant risk or protective factor in our sample. However, is was directly correlated with risk of neutropenia in others. ${ }^{13}$ Our sample was older than that of the Chilean registry, a possible explanation for the different risk of overall neutropenia in this study. Another possible explanation for the discrepancy is the different composition of ethnic groups between our population and the Chilean one. ${ }^{16}$ Specifically, benign ethnic neutropenia is more associated with African descent. ${ }^{21}$ The higher proportion of white non-Hispanics in our sample notwithstanding (88.7\%), the other $11.3 \%$ could explain the higher rate. Finally, socioeconomic factors (i.e., low educational levels) could be involved.

Our results must be interpreted considering the limitations of this study. The main constraint was limited data retrieval because of the query-based nature of collection. Although all patients on clozapine were accounted for, variables such as clozapine dose and other medications in use were scant. Certainty of antipsychotic use was obtained for $39.3 \%$ for the non-clozapine group, though more were assumed to be in use. However, all patients were derived from clinics caring for refractory psychotic, manic, and high-suicidality disorders, which allow us to assume that most were on antipsychotics or other psychiatric medications.

In conclusion, the present study provides consistent evidence in support of more flexible blood monitoring in those patients on clozapine with ANC $>2,000 / \mu \mathrm{L}$ during the first year of monitoring and in the absence of a severe medical condition (defined in this sample as HIV, HCV, or cancer). These results add compelling information from the South American population and could help guide clinical and public-health decisions towards flexibilizing blood monitoring guidelines for clozapine users.

\section{Disclosure}

The authors report no conflicts of interest.

\section{References}

1 Essali A, Haasan NA, Li C, Rathbone J. Clozapine versus typical neuroleptic medication for schizophrenia. Cochrane Database Syst Rev. 2009;2009(1): CD000059.

2 Li XB, Tang YL, Wang CY, de Leon J. Clozapine for treatmentresistant bipolar disorder: a systematic review. Bipolar Disord. 2015;17:235-47.

3 Wilby KJ, Johnson EG, Johnson HE, Ensom MH. Evidence-based review of pharmacotherapy used for Parkinson's disease psychosis. Ann Pharmacother. 2017;51:682-95.

4 Howes OD, McCutcheon R, Agid O, de Bartolomeis A, van Beveren NJ, Birnbaum ML, et al. Treatment-resistant schizophrenia: treatment response and resistance in psychosis (TRRIP) working group consensus guidelines on diagnosis and terminology. Am J Psychiatry. 2017; 174:216-29.

5 Meltzer HY, Alphs L, Green Al, Altamura AC, Anand R, Bertoldi A, et al. Clozapine treatment for suicidality in schizophrenia: international suicide prevention trial (InterSePT). Arch Gen Psychiatry. 2003;60:82-91.

6 Vermeulen JM, van Rooijen G, van de Kerkhof MP, Sutterland AL, Correll CU, de Haan L. Clozapine and long-term mortality risk in patients with schizophrenia: a systematic review and meta-analysis of studies lasting 1.1-12.5 years. Schizophr Bull. 2019;45:315-29. 
7 Thien K, Bowtell M, Eaton S, Bardell-Williams M, Downey L, Ratheesh A, et al. Clozapine use in early psychosis. Schizophr Res. 2018;199:374-9.

8 Crilly J. The history of clozapine and its emergence in the US market: a review and analysis. Hist Psychiatry. 2007;18:39-60.

9 Honigfeld G, Arellano F, Sethi J, Bianchini A, Schein J. Reducing clozapine-related morbidity and mortality: 5 years of experience with the Clozaril National Registry. J Clin Psychiatry. 1998;59 Suppl 3:3-7.

10 Nielsen J, Young C, Ifteni P, Kishimoto T, Xiang YT, Schulte PF, et al. Worldwide differences in regulations of clozapine use. CNS Drugs. 2016;30:149-61.

11 Clozapine Rems. Clozapine REMS Program [Internet]. 2015 [cited 2021 Jul 29]. www.clozapinerems.com/CpmgClozapineUl/home.u

12 Silveira AS, Rocha DM, Attux CR, Daltio CS, da Silva LA, Elkis H, et al. Patterns of clozapine and other antipsychotics prescriptions in patients with treatment-resistant schizophrenia in community mental health centers in São Paulo, Brazil. Arch Clin Psychiatry (São Paulo). 2015;42:165-70.

13 Myles N, Myles H, Xia S, Large M, Kisely S, Galletly C, et al. Meta-analysis examining the epidemiology of clozapine-associated neutropenia. Acta Psychiatr Scand. 2018;138:101-9.

14 Myles N, Myles H, Xia S, Large M, Bird R, Galletly C, et al. A metaanalysis of controlled studies comparing the association between clozapine and other antipsychotic medications and the development of neutropenia. Aust N Z J Psychiatry. 2019;53:403-12.
15 Inada K, Oshibuchi H, Ishigooka J, Nishimura K. Analysis of clozapine use and safety by using comprehensive national data from the Japanese clozapine patient monitoring service. J Clin Psychopharmacol. 2018;38:302-6.

16 Mena $\mathrm{Cl}$, Nachar RA, Crossley NA, González-Valderrama AA Clozapine-associated neutropenia in Latin America: incidence report of 5380 Chilean users. Int Clin Psychopharmacol. 2019;34: 257-63.

17 Siskind D, Honer WG, Clark S, Correll CU, Hasan A, Howes O, et al. Consensus statement on the use of clozapine during the COVID-19 pandemic. J Psychiatry Neurosci. 2020;45:222-3.

18 Balda M V, Garay OU, Papale RM, Bignone I, Bologna VG, Brandolini A, et al. Clozapine-associated neutropenia and agranulocytosis in Argentina (2007-2012). Int Clin Psychopharmacol. 2015;30:109-14.

19 Bachmann CJ, Aagaard L, Bernardo M, Brandt L, Cartabia M, Clavenna A, et al. International trends in clozapine use: a study in 17 countries. Acta Psychiatr Scand. 2017;136:37-51.

20 Tiihonen J, Lönnqvist J, Wahlbeck K, Klaukka T, Niskanen L, Tanskanen A, et al. 11-year follow-up of mortality in patients with schizophrenia: a population-based cohort study (FIN11 study). Lancet. 2009;374:620-7.

21 Shoenfeld Y, Alkan ML, Asaly A, Carmeli Y, Katz M. Benign familial leukopenia and neutropenia in different ethnic groups. Eur J Haematol. 1988;41:273-7. 